\title{
Mitonuclear epistasis and mitochondrial disease
}

Edward H. Morrow ${ }^{1}$, M. Florencia Camus ${ }^{2}$

${ }^{1}$ School of Life Sciences, University of Sussex, Brighton, BN1 9QG

2 Department of Genetics, Evolution and Environment, University College London, London, WC1E 6BT

Wang et al. ${ }^{1}$ report the joint effect of genetic variants in both the nuclear and mitochondrial DNA (mtDNA) on the occurrence of Kallman Syndrome in a large Han Chinese family. The nuclear variant (KAL1 c.146G>T, p.Cys49Phe) is not expected to cause changes to protein structure or function. Although the pathogenicity of the mitochondrial variant $\left(t R N A^{c y s}, \mathrm{~m} .5800 \mathrm{~A}>\mathrm{G}\right)$ has been predicted $^{2}$, there is no empirical evidence to support that prediction and it was not found to depress cellular oxidative phosphorylation ${ }^{1}$. Their interpretation is that the two point mutations act synergistically, causing abnormal migration of gonadotropin-releasing hormone neurons, which is thought to be the underlying mechanism for this developmental disorder. Since mutations in both genomes are required for manifestation of the phenotype, their results challenge how mitochondrial disease may be defined. We suggest that this two-locus/genome model of mitochondrial disease phenotypes may apply more broadly than is currently appreciated. Although the action of genetic background or modifiers have been implicated in altering the penetrance of primary pathological mutations underlying mitochondrial disease, reviews of this evidence have focused on specific disease sub-types ${ }^{3}$. Here, a review of the published evidence from the medical literature suggest that mitonuclear epistatic interactions are widespread and make a significant contribution to the variability in disease penetrance, which is a widely reported feature of mitochondrial pathologies ${ }^{4}$. We have identified 15 loci in mtDNA where the pathogenic effect (spanning a number of different mitochondrial diseases) is dependent upon the nuclear background, specific nuclear polymorphic sites, or expression levels of nuclear genes (Supplementary Table). In most cases, the defect in the mtDNA can be modified by multiple different nuclear loci, although some 'master modifiers' appear capable of influencing multiple mtDNA mutations (e.g. VARS2, LARS2). A 
further 10 nuclear loci have been identified where mitochondrial haplotype (or variants) have modified the pathogenic phenotype, which includes type II diabetes and Alzheimer's disease as well as classical mitochondrial diseases. Only 6 of these loci co-localize to mitochondria. In one example, the deleterious effect of the m.5703G>A mutation in human cell lines disappeared after a period of time in culture ${ }^{5}$. However, replacement of the nuclear background (using cybrids) reintroduced the deleterious phenotype. These data support a compensatory model of evolution within the nuclear genome in response to the presence of deleterious mutations within the mitochondrial genome. Together these studies highlight the potential role of mitonuclear epistasis in the expression and penetrance of human mitochondrial disease. This body of

evidence also contradicts assertions made during the debate over the safety and efficacy of mitochondrial replacement therapies about the role of mitonuclear interactions on human phenotypes. It is clear from the evidence we review here that predicting the outcome of nuclear transfer into new mitochondrial backgrounds will be difficult ${ }^{6}$, and would require a more far more complete understanding of inter-genomic epistasis than we currently have.

We declare no competing interests.

\section{REFERENCES}

1. Wang, F. et al. Point mutations in KAL1 and the mitochondrial gene MTtRNAcys synergize to produce Kallmann syndrome phenotype. Sci. Rep. 5, 13050 (2015).

2. Bannwarth, S. et al. Prevalence of rare mitochondrial DNA mutations in mitochondrial disorders. J. Med. Genet. 50, 704-714 (2013).

3. Bénit, P., El-Khoury, R., Schiff, M., Sainsard-Chanet, A. \& Rustin, P. Genetic background influences mitochondrial function: modeling mitochondrial disease for therapeutic development. Trends Mol. Med. 16, 210-217 (2010).

4. Limongelli, A. et al. Variable penetrance of a familial progressive necrotising encephalopathy due to a novel tRNAIle homoplasmic mutation in the mitochondrial genome. J. Med. Genet. 41, 342-349 (2004). 
5. Hao, H., Morrison, L. E. \& Moraes, C. T. Suppression of a Mitochondrial tRNA Gene Mutation Phenotype Associated with Changes in the Nuclear Background. Hum. Mol. Genet. 8, 1117-1124 (1999).

6. Chinnery, P. F. \& Zeviani, M. Mitochondrial Matchmaking. N. Engl. J. Med. 375, 1894-1896 (2016). 